\section{Ejercicio físico en pacientes con diabetes e hipertensión: prevalencia de respondedores y no respondedores para mejorar factores de riesgo cardiometabólicos}

\author{
JOHNATTAN CANO-MONTOYA ${ }^{1, \mathrm{a}, \mathrm{e}}$, RODRIGO RAMÍREZ-CAMPILLO ${ }^{2, \mathrm{~b}, \mathrm{f}}$, \\ FARID SADE CALLES ${ }^{3}$, MIKEL IZQUIERDO $^{4, \mathrm{c}, \mathrm{f}}$, NICOLE FRITZ SILVA ${ }^{5, \mathrm{~d}, \mathrm{e}}$, \\ RICARDO ARTEAGA SAN MARTÍN ${ }^{5, \mathrm{a}, \mathrm{e}}$, CRISTIAN ÁLVAREZ
}

\section{Effects of a six weeks exercise training program for type 2 diabetes mellitus and hypertensive patients}

Background: There is a wide interindividual variability in the response to a period of exercise training. The science have reported that a minimum of participants could be non-responders for improving different health-related outcomes after training. Aim: To compare the effects of a 6-weeks exercise program on body composition, cardiovascular and metabolic outcomes patients with type 2 diabetes and hypertension. Material and Methods: Data from 23 trained subjects were used in a secondary analysis of the response to exercise. Of these, 14 were considered adherent to training and nine as non-adherent. Body mass, height, waist circumference, four skinfolds and their sum, blood pressure and plasma triglyceride levels were assessed before and after the training period. Results: Among adherent participants, significant reductions were observed in the sum of four skinfolds ( $30 \pm 7$ to $27 \pm 6 \mathrm{~mm}, p \leq 0.05$ ), systolic blood pressure $(133 \pm 18$ to $127 \pm 20 \mathrm{mmHg} ; p \leq 0.05)$ and plasma triglycerides $(125 \pm 58$ to $102 \pm 34 \mathrm{mg} / \mathrm{dL} ; p \leq 0.05)$. No changes were observed in weight or diastolic blood pressure. Among non-adherent participants, no changes of measured parameters were observed. Among adherent participants, $57 \%$ were considered as non-responders for waist circumference, $7 \%$ for the sum of skinfold thickness, 50\% for systolic blood pressure, 64\% for diastolic blood pressure and 57\% for plasma triglycerides. Conclusions: Participants with a good adherence to a 6-weeks exercise training program experienced overall improvement in body composition, blood pressure and plasma triglycerides. The prevalence of non-responders varied considerably among measured outcomes.

(Rev Med Chile 2018; 146: 693-701)

Key words: Exercise; Diabetes Mellitus, Type 2; Hypertension; Risk Factors.

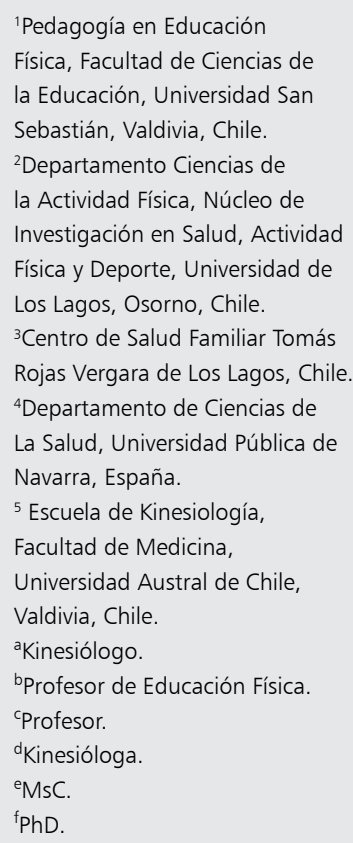

Conflicto de intereses: Todos los autores declaran que no existen conflictos de intereses entre las fuentes de financiamiento.

Recibido el 18 de agosto de 2017, aceptado el 26 de abril de 2018.

Correspondencia a: Cristian Álvarez Departamento de Ciencias de La Actividad Física, Universidad de Los Lagos, Osorno, Chile. Fono +56-64-2-333298 cristian.alvarez@ulagos.cl

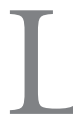
a diabetes mellitus tipo 2 (DT2) e hipertensión arterial (HTA) son enfermedades de alta prevalencia en Chile ${ }^{1}$ que están altamente asociadas a la inactividad física y sedentarismo ${ }^{2}$ donde el incremento la actividad física diaria, y mejora del fitness cardiorrespiratorio, se asocia a menores niveles de grasa corporal, presión arterial, y a una salud metabólica normal ${ }^{3}$. 
En este sentido, el ejercicio endurance (ejercicio de baja intensidad y larga duración), mejora el control glicémico ${ }^{4}$, reduce la grasa corporal ${ }^{5}$ y la presión $\operatorname{arterial}^{6} \mathrm{y}$ reduce los lípidos plasmáticos ${ }^{7}$. Sin embargo, dado que en la población no adhiere a estos ejercicios acusando falta de tiempo ${ }^{8}$, metodologías tiempo-efectivas ( $\leq 30 \mathrm{~min}$ por semana) como el ejercicio intermitente de alta intensidad de su sigla en inglés (HIIT) resultan en una alternativa eficiente para mejorar el control glicémico y reducir co-morbilidad asociada a personas de esta cohorte ${ }^{9,10}$.

A pesar de la evidencia existente del efecto positivo del ejercicio, normalmente expresada en la literatura en términos de "promedio", existe una amplia variabilidad interindividual al ejercicio ${ }^{11}$. Esto significa que mientras algunas personas presentan efectos benéficos, denominados respondedores (Rs), otros simplemente no presentan cambios o presentan una respuesta adversa después de ejercicio, denominados no respondedores $(\mathrm{NRs})^{12}$. Aunque factores genéticos ${ }^{13}, \mathrm{y}$ ambientales ${ }^{14-16}$ han sido escuetamente explorados, aún no se identifican los potenciales factores ambientales que dan cuenta de este fenómeno. Descartando la influencia genética que varía individualmente, no está del todo claro qué parámetros de salud, por ejemplo, composición corporal, presión arterial o marcadores metabólicos como los triglicéridos, presentan mayor o menor prevalencia de sujetos no respondedores en la población adherente a ejercicio.

Debido a que la adherencia es un factor clave para que el ejercicio surta efecto terapéutico para la DT2 e HTA ${ }^{17-20}$, y considerando que existen escasos reportes sobre la variabilidad interindividual al ejercicio HIIT, el objetivo del presente estudio fue comparar los efectos de un programa de 6 semanas de ejercicio de tipo HIIT en variables de composición corporal, cardiovasculares y metabólicas de pacientes con DT2 e HTA adherentes a ejercicio. Un segundo objetivo fue describir la prevalencia de Rs y NRs al ejercicio.

\section{Métodos}

\section{Diseño y muestra}

Estudio cuasi-experimental, y siguiendo análisis estadísticos secundarios de nuestro primer y original trabajo ${ }^{20}$. La captación de población se realizó mediante un llamado público invitando a participar de un programa de ejercicio físico HIIT de 6 semanas a adultos con diagnóstico de DT2 e HTA. Acudieron 23 sujetos adultos, 3 hombres y 20 mujeres, que cumplieron los criterios de inclusión: a) realizar $<150 \mathrm{~min} / \mathrm{semana}$ de actividad física de baja/moderada intensidad ${ }^{21} \mathrm{o}<75 \mathrm{~min} /$ semana de vigorosa intensidad ${ }^{22}$; b) diagnóstico clínico de DT2 e HTA con terapia farmacológica hipotensora o hipoglicemiante; c) control de salud actualizado y d) electrocardiograma de reposo normal. Los criterios de exclusión fueron: a) enfermedad ósea; b) enfermedad isquémica o arritmia y c) enfermedad pulmonar obstructiva crónica.

Previo y posterior a la intervención, se evaluaron parámetros de composición corporal, cardiovasculares y metabólicos. Finalizado el programa, los sujetos fueron asignados a un grupo adherente $(\mathrm{GA}, \mathrm{n}=14)$, considerando adherencia a las sesiones $\geq 70 \%$, o a un grupo no-adherente (GNA, $\mathrm{n}=9$ ) con adherencia $<70 \%$ siguiendo los criterios de estudios previos ${ }^{19}$. La muestra necesaria fue calculada de acuerdo a lo recomendado por estudios previos ${ }^{16} \mathrm{y}$ se describió la prevalencia (en porcentaje) de sujetos respondedores (Rs) y no respondedores (NRs). Todos los pacientes manifestaron su intención de participar en el estudio previa firma de un consentimiento informado y se consideró la Declaración de Helsinki de estudios con humanos, siendo aprobado por el comité de ética del Centro de Salud Familiar Tomas Rojas, Los Lagos, Chile. Las características de los pacientes se presentan en la Tabla 1.

\section{Respondedores (Rs), no-respondedores (NRs)}

Mediante criterios previamente utilizados ${ }^{12}$, la variabilidad interindividual al ejercicio fue categorizada como Rs y no respondedores NRs usando el error técnico (ET) de medición. Este ET fue calculado de acuerdo a los autores originales ${ }^{23}$, usando la fórmula: $\mathrm{ET}=D S_{\text {dif }} / \sqrt{2}$, donde DS es la varianza (desviación estándar) de las diferencias en los valores observados de 2 mediciones repetidas en cada test. Un NRs fue definido como un individuo quien demostró un incremento (en contra de cambios benéficos para la salud) que fuesen $\geq 2$ ET partiendo desde el valor "0". Así, un cambio a favor de efectos benéficos para la salud, que fue $<2$ ET significó la existencia de una alta probabilidad (12 a 1 Odds ratio) que la respuesta a ejercicio sea verdadera $^{23}$. El ET presentado en este estudio fue el siguiente; perímetro de cintura $1 \mathrm{~cm}$, sumatoria de 
4 pliegues 2,8 mm, PAS $8 \mathrm{mmHg}$, PAD $5 \mathrm{mmHg}$ y triglicéridos $20 \mathrm{mg} / \mathrm{dL}$.

\section{Actividad/inactividad física y composición corporal}

La primera semana todos los sujetos completaron un cuestionario breve (registro de los últimos 7 días de actividad física ${ }^{24}$ y y se registró el historial de vigencia en el programa de salud cardiovascular del Centro de Salud Familiar. En la segunda semana, se evaluó la composición corporal (peso, talla), el peso corporal y la talla se midió con una balanza con estadiómetro marca Detecto ${ }^{\mathrm{MR}}$ (modelo 3P7044, USA) con una precisión de $0,1 \mathrm{~kg}$ y $0,1 \mathrm{~cm}$ respectivamente. El índice de masa corporal (IMC) fue calculado en base al peso corporal dividido por la talla al cuadrado. Se evaluó el perímetro de cintura para estimar riesgo cardiovascular ${ }^{25}$ con una cinta métrica con precisión de $0,1 \mathrm{~cm}$. Se midió tejido adiposo subcutáneo a través de 4 pliegues de grasa subcutánea (tricípital, subescapular, suprailíaco y abdominal) con un Caliper Lange ${ }^{\mathrm{MR}}$ (Beta Technology Ind., Santa Cruz, California, USA).

\section{Presión arterial}

Asimismo, en la primera semana, la presión arterial sistólica (PAS) y diastólica (PAD) se midieron después de 15 min de reposo con un monitor digital marca OMRON ${ }^{M R}$ (modelo HEM-742 INT, USA), siguiendo criterios previamente utilizados y se clasificó la presión arterial de acuerdo a criterios ampliamente conocidos ${ }^{26}$.

\section{Medición plasmática}

En la segunda semana, se tomaron muestras plasmáticas para medir los niveles de triglicéridos en ayuna mediante un analizador químico marca Wiener Lab ${ }^{\mathrm{MR}}$ (modelo Metrolab 2300 plus, Argentina). Se extrajeron muestras de sangre venosa (aprox. 3,5 ml) fueron obtenidas después de $12 \mathrm{~h}$ de ayuno, depositándose estas en un tubo con gel separador y activador de coagulación, se centrifugaron a $4.000 \mathrm{rpm}$ por $10 \mathrm{~min}$ para obtención de suero, utilizándose el test enzimático colorimétrico mediante GPO-POD.

\section{Programa de ejercicio intermitente de alta inten- sidad (HIIT)}

En la tercera semana los sujetos fueron citados para dar inicio al proceso de familiarización al ejercicio HIIT por dos semanas (4 sesiones). En la sesión número 5, los sujetos iniciaron el programa HIIT, consistiendo en 1 min de pedaleo en bicicleta, 2 min de recuperación y ejecutado 10 veces $(1 \times 2 \times 10)^{1}$. Durante el protocolo $1 \times 2 \times 10$, se controló una intensidad de esfuerzo muscular del pedaleo de 8 a 10 de acuerdo a la escala de Borg modificada ${ }^{27}$, intensidad homologable a una frecuencia cardiaca cercana al máximo teori$\mathrm{Co}^{27}$, donde cada paciente podía cesar el ejercicio cuando así lo estimara, no siendo una imposición la finalización total de cada intervalo de esfuerzo. Cuando el paciente no se recuperaba (frecuencia cardíaca $>70 \%$ del máximo teórico según formula de la ACSM [220 - edad $]^{28}$ ) en el tiempo de pausa, se le permitía una mayor duración de descanso hasta lograr la recuperación cardiovascular. Cada 2 semanas se aumentó la carga de la bicicleta, debido a las adaptaciones fisiológicas y mejora del umbral de base del paciente, ejercitándose siempre el paciente en cada intervalo de esfuerzo en una intensidad muscular de 8 a 10 puntos según la escala de Borg modificada, utilizándose bicicletas marca LIFEFITNESS $^{\mathrm{MR}}$ (modelo C1, USA).

\section{Análisis estadísticos}

Se utilizó media \pm desviación estándar para describir las variables cuantitativas. Se utilizó el test de Shapiro-Wilk para determinar la normalidad de los datos y el test de Levene para establecer la homocedasticidad. Se aplicó el test t de Student para testear las diferencias en la línea de base de las variables. Posterior a la selección de sujetos quienes terminaron la intervención, se calculó las diferencias pre-post en delta porcentual en cada variable, para así proceder a la clasificación de la muestra del grupo adherente en Rs y NRs usando el criterio de $2 \mathrm{ET}^{12}$. La prevalencia de sujetos Rs y NRs se describió mediante porcentaje (\%) en el grupo adherente. El nivel para significancia estadística se estableció en $p \leq 0,05$. Todos los análisis estadísticos se realizaron utilizando el software SPSS $^{\mathrm{MR}}$ (versión 18.0).

\section{Resultados}

No se registraron diferencias significativas en la talla, el peso corporal, e índice de masa corporal antes de intervención, (Tabla 1). No se registraron cambios significativos en el peso corporal, e índice de masa corporal después de intervención, (Tabla 1). 
En el grupo GA, se presentó una reducción no significativa $(\mathrm{p}>0,05)$ del perímetro de cintura en promedio desde 101,5 $\pm 2,6$ a $100,4 \pm 2,2 \mathrm{~cm}$, (Figura 1, panel a). En el grupo GA, de un total de 14 sujetos, 6 fueron Rs para reducir el perímetro de cintura (43\%), y 8 fueron NRs (57\%), (Figura 1 , panel b). En tanto, el grupo GNA presentó un incremento no significativo en el perímetro de cintura desde 99,3 $\pm 3,4$ a 99,8 $\pm 3,5 \mathrm{~cm}$, (Figura 1, panel a).

Tabla 1. Características antropométricas de 2 grupos de sujetos con DT2 e HTA participantes de 6 semanas de ejercicio tipo HIIT

\begin{tabular}{|lllll|}
\hline Variables & Test & GA & GNA & Valor p \\
$\mathrm{n}=$ & & 14 & 9 & \\
Talla & & $1,56 \pm 0,08$ & $1,53 \pm 0,05$ & 0,189 \\
Peso $(\mathrm{kg})$ & Pre & $75,2 \pm 13$ & $71,4 \pm 15$ & 0,531 \\
& Post & $74,9 \pm 13$ & $71,6 \pm 15$ & 0,528 \\
& $\Delta \mathrm{kg}$ & $-0,3$ & $+0,2$ & \\
& $\Delta \%$ & $-0,3$ & $+0,2$ & 0,090 \\
IMC $\left(\mathrm{kg} / \mathrm{m}^{2}\right)$ & Pre & $30,9 \pm 3,9$ & $30,3 \pm 5,9$ & 0,079 \\
& Post & $30,8 \pm 3,8$ & $30,3 \pm 5,8$ & \\
& $\Delta \mathrm{kg}$ & $-0,1$ & 0 & \\
& $\Delta \%$ & $-0,3$ & 0 & \\
\hline
\end{tabular}

Valores de variables son presentados como media \pm DE. Grupos son presentados como grupo adherente (GA) y grupo no-adherente (GNA). (IMC): índice de masa corporal. Diferencias entre variables continuas fueron determinadas con t-test para muestras independientes.

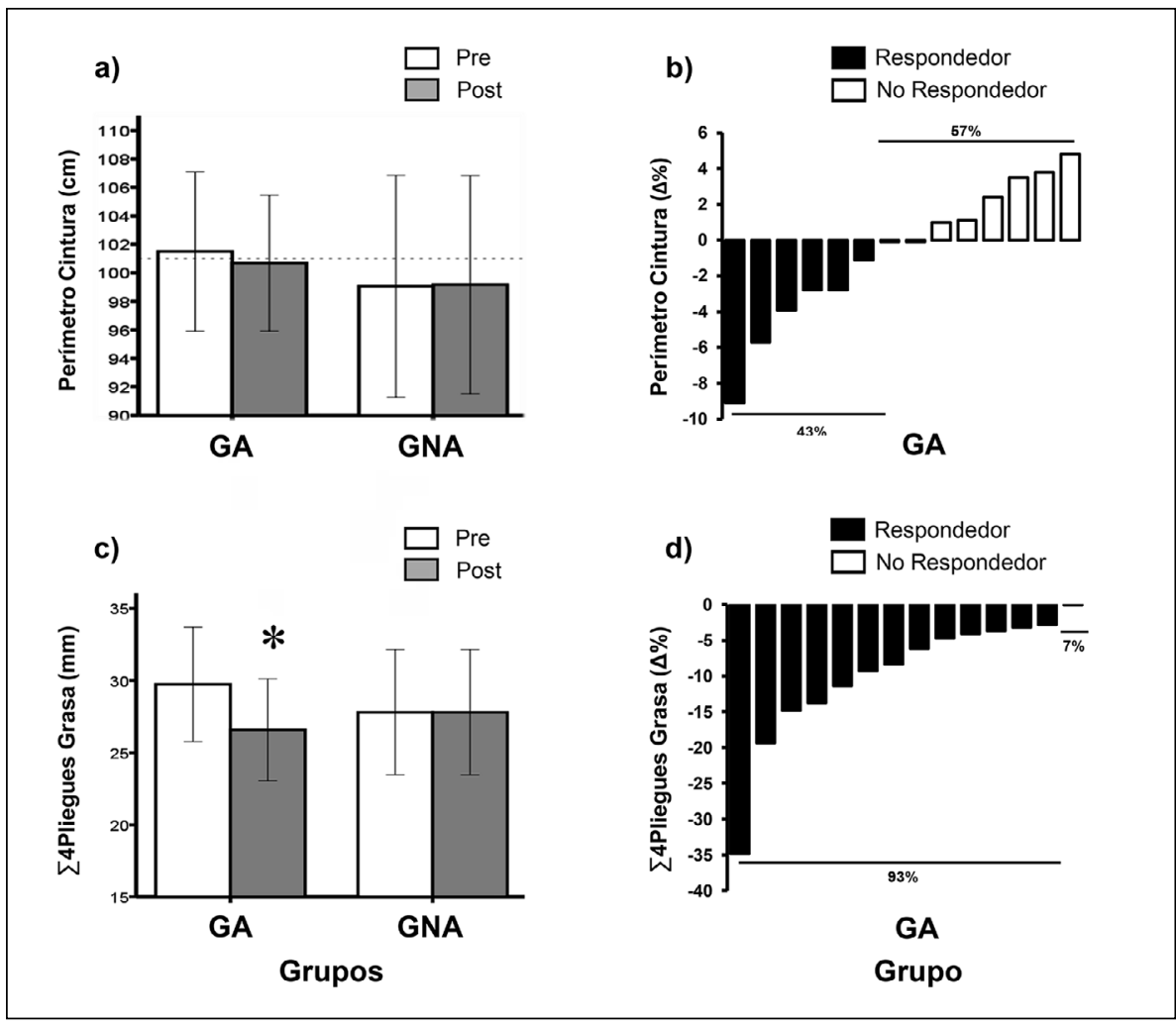

Figura 1. Cambios promedio (panel a y c) y cambios individuales (panel b y d) en la composición corporal de 2 grupos de sujetos con DT2 e HTA. Grupos son descritos como: (GA) grupo adherente, (GNA) grupo no-adherente. Prevalencia de sujetos respondedores y no respondedores se presenta como porcentaje (\%). *Indica cambio significativos pre-post a nivel $p<0,05$. 
En el grupo GA, la sumatoria de 4 pliegues de grasa ( $\sum 4$ Pliegues) se redujo significativamente $(\mathrm{p} \leq 0,05)$ desde $30 \pm 7$ a $27 \pm 6 \mathrm{~mm}$ post intervención, (Figura 1, panel c). De acuerdo con los cambios individuales, en este grupo de un total de 14 sujetos, 13 (93\%) fueron Rs, y 1 fue NRs (7\%), (Figura 1, panel d).

En el grupo GA se presentó una reducción significativa $(\mathrm{p} \leq 0,05)$ de la PAS desde $133 \pm 18$ a $127 \pm 20 \mathrm{mmHg}$ post intervención, (Figura 2, panel a). Considerando sólo el grupo GA, de un total de 14 sujetos, 7 (50\%) fueron Rs, y 7 (50\%) fueron NRs, (Figura 2, panel b). En tanto el grupo GNA, presento una reducción no significativa de la PAS,
(Figura 2, panel b).

Por otra parte, en el mismo grupo GA, la PAD, presentó un incremento no significativo, similar al grupo GNA, (Figura 2, panel c). En tanto, en el grupo GA, de un total de 14 sujetos, 5 (36\%) fueron Rs, y 9 (64\%) NRs, (Figura 2, panel c).

A nivel metabólico, los niveles de triglicéridos plasmáticos se redujeron significativamente $(\mathrm{p} \leq 0,05)$ desde $125 \pm 58$ a $102 \pm 34 \mathrm{mg} / \mathrm{dl}$, en el grupo GA (Figura 3, panel a). En esta variable, de un total de 14 sujetos, 6 (43\%) fueron Rs, y $8(57 \%)$ fueron NRs, (Figura 3, panel b). En el grupo GNA en tanto, no se presentaron cambios estadísticamente significativos.

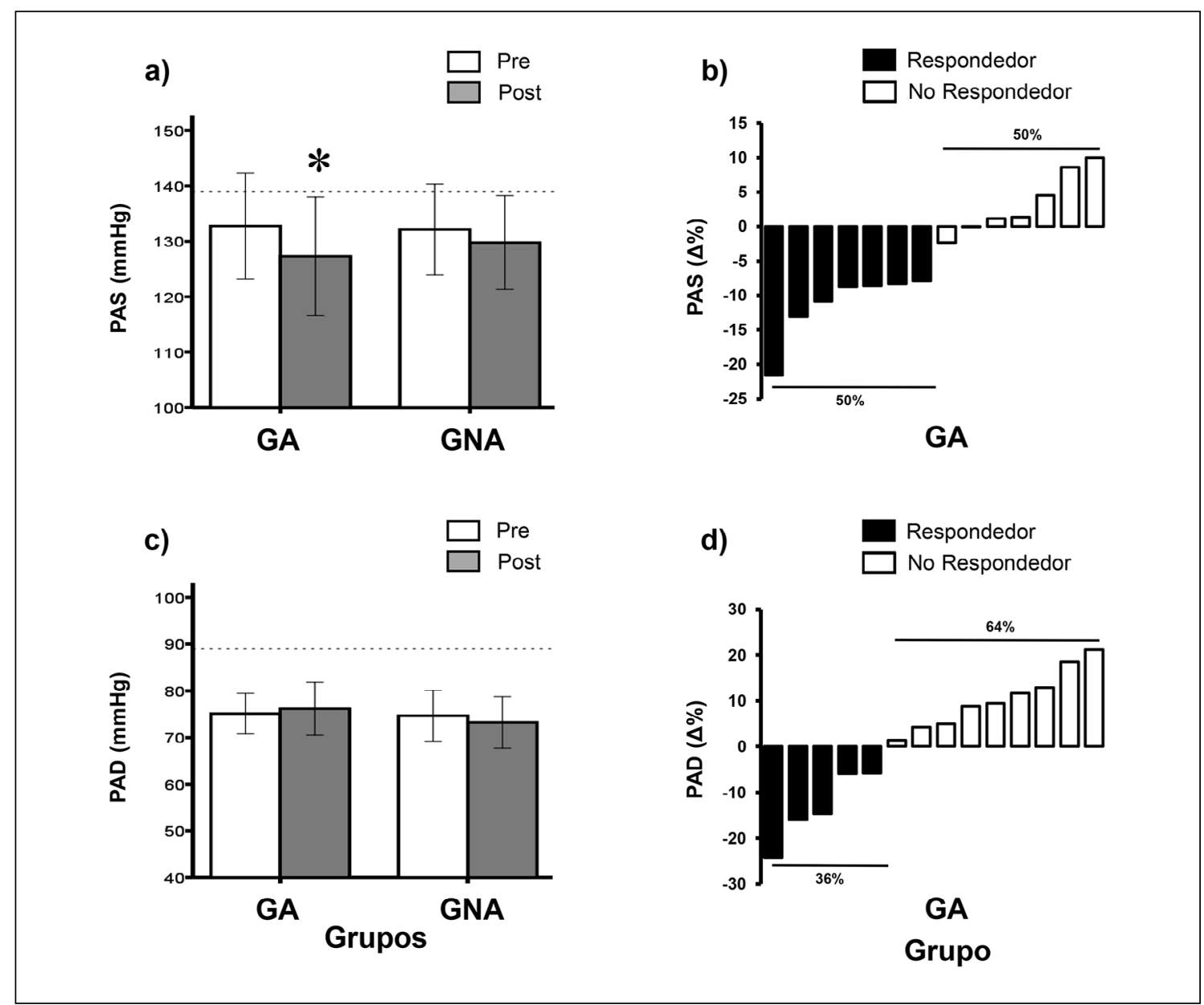

Figura 2. Cambios promedio (panel a y c) y cambios individuales (panel b y d) en la presión arterial sistólica y diastólica de 2 grupos de sujetos con DT2 e HTA. Grupos son descritos como: (GA) grupo adherente, (GNA) grupo no-adherente. Prevalencia de sujetos respondedores y no respondedores se presenta como porcentaje (\%). *Indica cambio significativos pre-post a nivel $p<0,05$. 


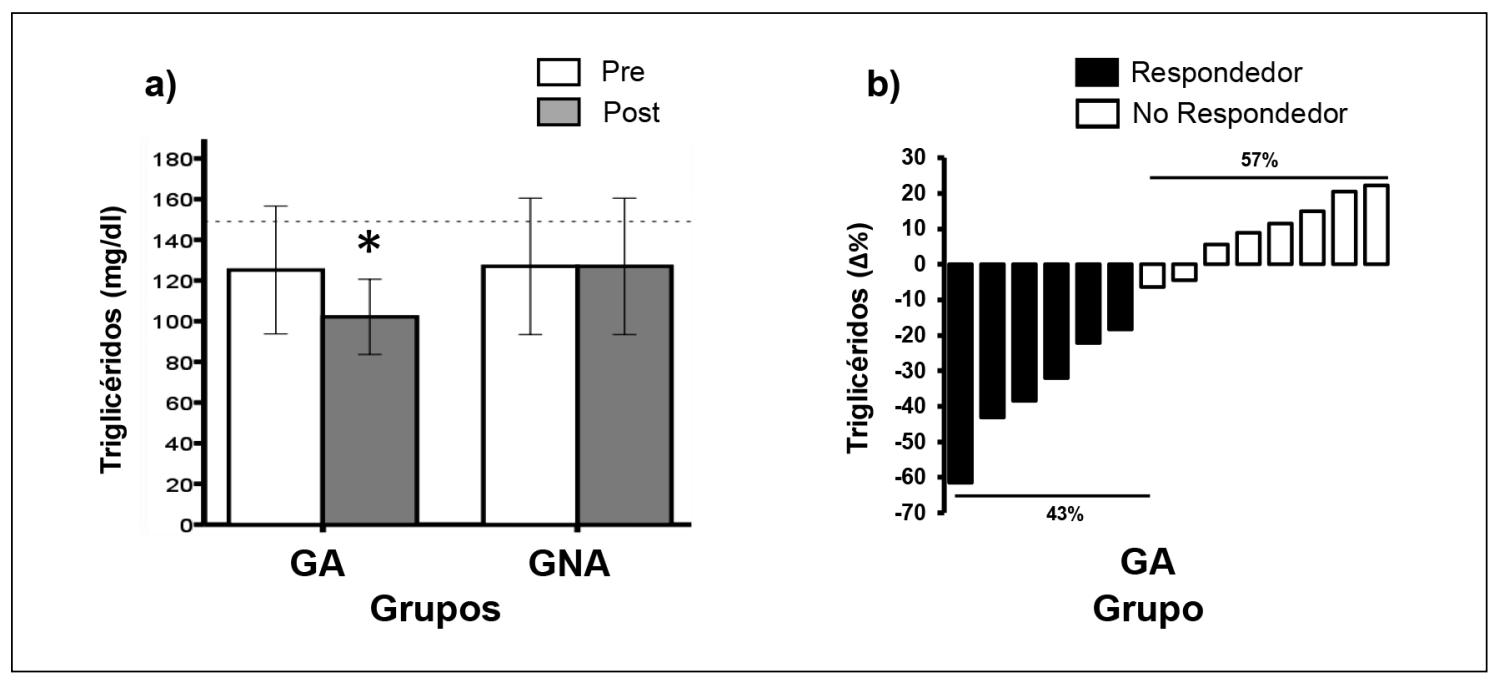

Figura 3. Cambios promedio (panel $\mathbf{a}$ y $\mathbf{c}$ ) y cambios individuales (panel $\mathbf{b}$ y $\mathbf{d}$ ) en los niveles de triglicéridos plasmáticos de 2 grupos de sujetos con DT2 e HTA. Grupos son descritos como: (GA) grupo adherente, (GNA) grupo no-adherente. Prevalencia de sujetos respondedores y no respondedores se presenta como porcentaje (\%). ${ }^{\star}$ Indica cambio significativos pre-post a nivel $p<0,05$.

\section{Discusión}

Los mayores hallazgos del presente estudio indican que cuando los pacientes son adherentes a HIIT se i) reduce la grasa subcutánea, la presión sistólica y los niveles de triglicéridos, existiendo también ii) una mayor prevalencia de sujetos Rs en la reducción de grasa subcutánea, y una mayor prevalencia de sujetos NRs en las variables PAS, PAD, y triglicéridos. Así, en programas cortos de HIIT, los mayores efectos benéficos provienen de la reducción en la grasa corporal, y que existe una amplia variabilidad interindividual para otros parámetros de composición corporal, cardiovascular y metabólico, donde independiente de los efectos benéficos y significativos en "promedio", alrededor del $\sim 50 \%$ de sujetos podrían ser NRs.

La mejora de parámetros de composición corporal en pacientes con DT2 o HTA no es nue$\mathrm{va}^{19,29}$. Mancilla y cols. ${ }^{19}$ redujeron $-4,2 \mathrm{~kg}$ de grasa después de 12 semanas de HIIT en pacientes con DT2. Molina y cols. ${ }^{29}$ mostraron que 12 sesiones de HIIT reducen $-1,8 \mathrm{~kg}$ de grasa en mujeres y $-3,5 \mathrm{~kg}$ en hombres. Después de un entrenamiento de fuerza de 12 semanas Churchward-Venne y cols. ${ }^{31}$ reportaron un $4,5 \%$ de NRs, mientras un similar programa del doble de duración (24 semanas) produjo $23 \%$ de prevalencia de NRs para reducir el peso corporal. Aunque, se debe tener presente que el ejercicio de fuerza per se produce incrementos en la masa muscular y por ende en el peso corporal total. En cuanto a la reducción de grasa subcutánea, Tremblay y cols. ${ }^{31}$ a través de un protocolo de HIIT en personas inactivas, observaron una reducción de $\sum 4$ Pliegues $(-2,75$ $\mathrm{mm})$, similar al presente estudio, sin embargo el tiempo de ejercicio de estos autores fue superior (15 semanas).

Aunque la literatura es muy amplia, ya han sido difundidos los efectos de diferentes programas de ejercicio en pacientes con $\mathrm{HTA}^{32}$, o con DT2 y $\mathrm{HTA}^{20}$. Un reciente estudio de revisión concluyó que intervenciones entre 4-12 semanas de HIIT reducen $-0,3 \mathrm{mmHg}$ la $\mathrm{PAS}^{33}$. Luego de 5 meses de ejercicio endurance $(65-80 \%$ consumo de oxígeno máximo pico, caminando), o en ejercicio de fuerza (8-12 repeticiones por serie, 8 ejercicios, $70-85 \%$ of 1 repetición máxima), o la combinación de ambos, Moker y cols. ${ }^{34}$ reportaron $\sim 60 \%$ de NRs para reducir la PAS. En contraste, en el presente estudio nosotros reportamos que una prevalencia de $50 \%$ de sujetos NRs para reducir la PAS, y 64\% de NRs para reducir la PAD (Figura 2, panel b y d). Posterior a un programa de HIIT (6 semanas), Higgins y cols. ${ }^{35}$ reportaron una prevalencia de NRs de un 
$\sim 60 \%$ para reducir la PAS y PAD. Bouchard y cols. ${ }^{11}$ después de 6 meses de ejercicio endurance realizado en 5 diferentes países reportaron una prevalencia entre 5 y $30 \%$ de sujetos NRs para reducir la PAS. En cuanto a las posibles causas de no respuesta al ejercicio Bouchard y cols. ${ }^{36}$ reportaron que la presión arterial sistólica de base $(<145 \mathrm{mmHg}$ vs $>145 \mathrm{mmHg})$ presentaría una directa relación con el porcentaje de NRs al ejercicio siendo esta mayor (35\% versus $15 \%$ ) en los sujetos con niveles menores de PAS.

Aunque la evidencia del HIIT es amplia mejorando el control glicémico ${ }^{4,7,9}$, en la reducción de lípidos no lo es tanto. Nosotros observamos una prevalencia de NRs de 57\% en la reducción de TG (Figura 3, panel b). En estudios de mayor duración mediante caminata diaria durante 12 meses, Yates y cols. ${ }^{37}$ mostraron incluso una respuesta adversa de $21 \%$ de la muestra. Bouchard y cols. ${ }^{11}$, después de 6 meses de ejercicio endurance mostraron una respuesta adversa de $13,1 \%$ en la reducción de TG. Parte de las diferencias en la prevalencia de NRs entre estudios, podrían ser explicadas por la frecuencia, volumen, intensidad de ejercicio.

Algunas fortalezas del presente estudio fueron que se reportaron variables de composición corporal, cardiovascular y de tipo metabólico, mostrando los efectos y la prevalencia de Rs y NRs, y se utilizó un número de muestra frecuentemente utilizado en intervenciones con ejercicio ( $\sim 10-20$ sujetos). Algunas debilidades fueron la existencia de disparidad en la muestra en cuanto al sexo, recomendándose para futuros estudios separar el efecto por género, y no se controlaron los patrones de actividad física ni dieta antes y posterior a ejercicio, sin embargo, se recordó a los participantes de mantener los mismos patrones declarados inicialmente.

Esta información podría ser útil para elegir qué programas promueven una menor cantidad de NRs antes de pretender buscar beneficios en diferentes parámetros de salud, y así los profesionales de las ciencias de la salud y la actividad física tener cierta capacidad de predicción de las respuestas cuando los pacientes son adherentes. En conclusión, seis semanas de ejercicio HIIT mejoran la composición corporal, presión arterial y triglicéridos de pacientes diabéticos e hipertensos adherentes a ejercicio, existiendo una menor prevalencia de NRs en la reducción de grasa subcutánea.
Agradecimientos: Queremos agradecer al personal del Hospital Público de Los Lagos en especial a su directora Ana María Gayoso, tecnólogos médicos Amanda Piel, Alan Cofré. También agradecemos al Dr Gonzalo Gajardo del Laboratorio de Genética, Acuicultura \& Biodiversidad de la Universidad de Los Lagos, por su colaboración en la revisión del trabajo, así como finalmente a los participantes de este estudio por su compromiso con el programa de ejercicio.

\section{Referencias}

1. MINSAL. Ministerio de Salud. Gobierno de Chile. Encuesta Nacional de Salud ENS Chile 2009-2010. Disponible en: http://www.redsalud.gov.cl/portal/url/ item/99c12b89738d80d5e04001011e0113f8.pdf 2011.

2. Celis-Morales C, Salas C, Sanzana R, Martínez M, Leiva A, Diaz X, et al. Socio-demographic patterning of physical activity levels and sedentary behaviour in Chile: results from the National Health Survey. III World Conference in Public Health Nutrition. J Public Health (Oxf) 2016; 38 (2): e98-e105.

3. Cristi-Montero C, Ramírez-Campillo R, Álvarez C, Garrido Méndez A, Martínez MA, Díaz Martínez X, et al. Fitness cardiorrespiratorio se asocia a una mejora en marcadores metabólicos en adultos chilenos. Rev Med Chile 2016; 144 (8): 980-9.

4. Sigal RJ, Kenny GP, Boulé NG, Wells GA, Prud'homme D, Fortier M, et al. Effects of Aerobic Training, Resistance Training, or Both on Glycemic Control in Type 2 Diabetes A Randomized Trial. Ann Intern Med 2007; 147 (6): 357-69.

5. Riesco E, Tessier S, Perusse F, Turgeon S, Tremblay A, Weisnagel J, et al. Impact of walking on eating behaviors and quality of life of premenopausal and early postmenopausal obese women. Menopause 2010; 17 (3): 52938.

6. Taylor-Tolbert NS, Dengel DR, Brown MD, McCole SD, Pratley RE, Ferrell RE, et al. Ambulatory blood pressure after acute exercise in older men with essential hypertension. Am J Hypertens 2000; 13 (1 Pt 1): 44-51.

7. Snowling NJ, Hopkins WG. Effects of Different Modes of Exercise Training on Glucose Control and Risk Factors for Complications in Type 2 Diabetic Patients: A meta-analysis. Diabetes Care 2006; 29 (11): 2518-27.

8. Anderson CB. When more is better: number of motives and reasons for quitting as correlates of physical activity in women. Health Educ Res 2003; 18 (5): 525-37.

9. Little JP, Gillen JB, Percival ME, Safdar A, Tarnopols- 
ky MA, Punthakee Z, et al. Low-volume high-intensity interval training reduces hyperglycemia and increases muscle mitochondrial capacity in patients with type 2 diabetes. J Appl Physiol 1985; 2011; 111 (6): 1554-60.

10. Ciolac EG. High-intensity interval training and hypertension: maximizing the benefits of exercise? Am J Cardiovasc Dis 2012; 2 (2): 102-10.

11. Bouchard C, Blair SN, Church TS, Earnest CP, Hagberg JM, Häkkinen $\mathrm{K}$, et al. Adverse metabolic response to regular exercise: is it a rare or common occurrence? PLoS One 2012; 7 (5): e37887.

12. Bonafiglia JT, Rotundo MP, Whittall JP, Scribbans TD, Graham RB, Gurd BJ. Inter-Individual Variability in the Adaptive Responses to Endurance and Sprint Interval Training: A Randomized Crossover Study. PLoS One 2016; 11 (12): e0167790.

13. Timmons JA. Variability in training-induced skeletal muscle adaptation. J Appl Physiol (1985) 2011; 110 (3): 846-53.

14. Álvarez C, Ramírez-Campillo R, Ramírez-Vélez R, Izquierdo M. Prevalence of Non-Responders In Glucose Control Markers After 10-Weeks of High-Intensity Interval Training in Higher and Lower Insulin Resistant Adult Women. Front Physiol 2017; 8: 479.

15. Álvarez C, Ramírez-Campillo R, Ramírez-Vélez R, Izquierdo M. Effects of 6-Weeks High-Intensity Interval Training in Schoolchildren with Insulin Resistance: Influence of Biological Maturation on Metabolic, Body Composition, Cardiovascular and Performance Non-responses. Front Physiol 2017; 8: 444.

16. Álvarez C, Ramírez-Campillo R, Ramírez-Vélez R, Izquierdo $\mathrm{M}$. Effects and prevalence of nonresponders after 12 weeks of high-intensity interval or resistance training in women with insulin resistance: a randomized trial. J Appl Physiol (1985) 2017; 122 (4): 985-96.

17. Álvarez C, Ramírez-Campillo R, Henríquez-Olguín C, Castro-Sepúlveda M, Carrasco V, Martínez C. ¿Pueden ocho semanas de ejercicio físico combinado normalizar marcadores metabólicos de sujetos hiperglicémicos y dislipidémicos?. Rev Med Chile 2014; 142 (4): 458-66.

18. Álvarez C, Ramírez-Campillo R, Martínez-Salazar C, Mancilla R, Flores-Opazo M, Cano-Montoya J, et al. Low-Volume High-Intensity Interval Training as a Therapy for Type 2 Diabetes. Int J Sports Med 2016; 37 (9): 723-9.

19. Mancilla R, Torres P, Álvarez C, Schifferli I, Sapunar J, Diaz E. High intensity interval training improves glycemic control and aerobic capacity in glucose intolerant patients. Rev Med Chile 2014; 142 (1): 34-9.

20. Cano-Montoya J, Álvarez C, Martínez C, Salas A, Sade
F, Ramírez-Campillo R. Cardiovascular recovery during intermittent exercise in highly-adherent participants with hypertension and type 2 diabetes mellitus. Rev Med Chile 2016; 144 (9): 1150-8.

21. Garber CE, Blissmer B, Deschenes MR, Franklin BA, Lamonte MJ, Lee I-M, et al. Quantity and Quality of Exercise for Developing and Maintaining Cardiorespiratory, Musculoskeletal, and Neuromotor Fitness in Apparently Healthy Adults: Guidance for Prescribing Exercise. Med Sci Sports Exerc 2011; 43 (7): 1334-59.

22. O'Donovan G, Blazevich AJ, Boreham C, Cooper AR, Crank H, Ekelund U, et al. The ABC of Physical Activity for Health: a consensus statement from the British Association of Sport and Exercise Sciences. J Sports Sci 2010; 28 (6): 573-91.

23. Hopkins WG. Measures of Reliability in Sports Medicine and Science. Sports Med 2000; 30 (1): 1-15.

24. Lee PH, Macfarlane DJ, Lam T, Stewart SM. Validity of the international physical activity questionnaire short form (IPAQ-SF): A systematic review. Int J Behav Nutr Phys Act 2011; 8: 115.

25. Lean ME, Han TS, Morrison CE. Waist circumference as a measure for indicating need for weight management. BMJ 1995; 311 (6998): 158-61.

26. Chobanian AV, Bakris GL, Black HR, Cushman WC, Green LA, Izzo JL, et al. Seventh report of the joint national committee on prevention, detection, evaluation, and treatment of high blood pressure. Hypertension 2003; 42 (6): 1206-52.

27. Ciolac EG, Mantuani SS, Neiva CM, Verardi C, Pessôa-Filho DM, Pimenta L. Rating of perceived exertion as a tool for prescribing and self regulating interval training: a pilot study. Biol Sport 2015; 32 (2): 103-8.

28. American College de Sport Medicine. ACSM's Guidelines for Exercise Testing and Prescription. $6^{\text {th }}$ ed. Baltimore, MD: Lippincott WILLIAMS \& Wilkins, 2000.

29. Molina C, Cifuentes G, Martínez C, Mancilla R, Díaz E. Effects of 12 sessions of high intensity intermittent training and nutrition counseling on body fat in obese and overweight participants. Rev Med Chile 2016; 144 (10): 1254-9.

30. Churchward-Venne TA, Tieland M, Verdijk LB, Leenders M, Dirks ML, de Groot LC, et al. There are no nonresponders to resistance-type exercise training in older men and women. J Am Med Dir Assoc 2015; 16 (5): 400-11.

31. Tremblay A, Simoneau JA, Bouchard C. Impact of exercise intensity on body fatness and skeletal muscle metabolism. Metabolism 1994; 43 (7): 814-8.

32. Pescatello LS, MacDonald HV, Lamberti L, Johnson BT. Exercise for hypertension: a prescription update 
Ejercicio físico en pacientes con diabetes e hipertensión - J. Cano-Montoya et al

integrating existing recommendations with emerging research. Curr Hypertens Rep 2015; 17 (11): 87.

33. García-Hermoso A, Cerrillo-Urbina A, Herrera-Valenzuela T, Cristi-Montero C, Saavedra J, Martínez-Vizcaíno V. Is high-intensity interval training more effective on improving cardiometabolic risk and aerobic capacity than other forms of exercise in overweight and obese youth? A meta-analysis. Obes Rev 2016; 17 (6): 531-40.

34. Moker EA, Bateman LA, Kraus WE, Pescatello LS. The Relationship between the Blood Pressure Responses to Exercise following Training and Detraining Periods. PloS one 2014; 9 (9): e105755.
35. Higgins TP, Baker MD, Evans S-A, Adams RA, Cobbold C. Heterogeneous responses of personalised high intensity interval training on type 2 diabetes mellitus and cardiovascular disease risk in young healthy adults. Clin Hemorheol Microcirc 2015; 59 (4): 365-77.

36. Bouchard $\mathrm{C}$, Rankinen $\mathrm{T}$. Individual differences in response to regular physical activity. Med Sci Sports Exerc 2001; 33 (6 Suppl): S446-51; discussion S52-3.

37. Yates T, Davies MJ, Edwardson C, Bodicoat DH, Biddle SJ, Khunti K. Adverse responses and physical activity: secondary analysis of the PREPARE trial. Med Sci Sports Exerc 2014; 46 (8): 1617-23. 